\title{
A new Neighborhood Structure and its fast Evaluation Strategy in using Iterated Local Search to Solve Single Machine Scheduling
}

\author{
Hongyun $\mathrm{Xu}$, Quan OuYang ${ }^{+}$ \\ School of Mathematics \& Computer Science, JiangHan University, Wuhan, China
}

\begin{abstract}
In this paper, a new neighborhood structure called Block Exchange and its fast evaluation strategy are proposed when using the Iterated Local Search (ILS) algorithm to solve the single machine scheduling problem without setup times. Applying the proposed ILS algorithm which adopts the Block Exchange neighborhood and fast evaluation strategy to solve 125 public benchmark instances, we achieve highly competitive results compared with the other two ILS algorithms adopt two neighborhoods widely used in the previous literature for single machine scheduling, respectively. Specifically, ILS with Block Exchange neighborhood and fast evaluation strategy finds the best-known results for all the 125 instances, while the other two ILS algorithms can not obtain the best-known results for 97 and 92 instances. The experimental results show that the proposed Block Exchange neighborhood and its fast evaluation strategy are highly effective and efficient.
\end{abstract}

Keywords: single machine scheduling, neighborhood structure, Iterated Local Search, Block Exchange.

\section{Introduction}

The single machine scheduling problem without setup times studied in this paper can be described as follows: Suppose $N$ is the total number of jobs to be processed in one machine. Each job $j$ has a processing time $p_{j}$, a weight $w_{j}$, and a due date $d_{j}$. The start time of each job is the completion time of the previous job in the job sequence. Using $\pi=\left\{\pi_{1}, \pi_{2}, \pi_{3}, \ldots, \pi_{N}\right\}\left(\pi_{i} \neq \pi_{j}, i \neq j\right)$ to represent a processing sequence, where $\pi_{j}$ denotes the $j$-th job in the entire processing sequence and its completion time is denoted by $C_{j}, \Delta f\left(\pi_{j}\right)$. The tardiness of the $\pi_{j}$ is $T_{j}, T_{j}=\max \left\{C_{j}-d_{\pi_{j}}, 0\right\}$. Then the total tardiness of $\pi$ is denoted by $T, T=\sum w_{j} T_{j}$. So, the single machine scheduling problem without setup times can be denoted as $1 \| \sum w_{j} T_{j}$, a specíal case of $1\left|s_{i j}\right| w_{j} T_{j}$, has been shown to be strongly NP-hard [1],[2]. The $1 \| \sum w_{j} T_{j}$ problem is to find a job sequence that minimizes $T$.

There are several heuristic and metaheuristic algorithms, for instance, particle swarm optimization algorithm, tabu search algorithm, and variable neighborhood search, developed to solve the $1 \| \sum w_{j} T_{j}$ problem in [3]-[5].

\section{Iterated Local Search algorithm}

Iterated Local Search (ILS) is a simple but widely used heuristic algorithm. It uses the local search technique and the perturbation mechanism to search the solution space. The local search technique makes the current solution could be converged to the local extreme. The perturbation mechanism is responsible for jumping out of the local optimal to reach a new search space. The ILS algorithm consists of four parts: [1] Generate Initial Solution: The initial solution is usually composed of some constructive heuristic algorithm or randomly generated; [2] Local Search; [3] Perturbation: the method to jump out of the local optimal; [4]

\footnotetext{
+ Corresponding author. Tel.: +86 18086623297.

E-mail address: oyq8888@163.com (Quan OuYang).
} 
Acceptance Criterion: the algorithm stops when the certain conditions met. The basic framework of the iterated local search algorithm is shown as follows:

Step 1: $\mathrm{s} \leftarrow$ generate initial solution

Step 2: s'Ł local search (s)

Step 3: repeat

Step 4: $\mathrm{s}^{*} \leftarrow$ perturbation $\left(\mathrm{s}^{\prime}\right)$

Step 5: $\mathrm{s}^{* \prime} \leftarrow$ local search $\left(\mathrm{s}^{*}\right)$

Step 6: $\mathrm{s}^{\prime} \leftarrow$ acceptance criterion $\left(\mathrm{s}^{\prime}, \mathrm{s}^{* \prime}\right)$

Step 7: until stop condition met

In our ILS algorithm, the initial solution is generated randomly. At the end of each round of local search, if $\mathrm{s}^{* \prime}$ is better than $\mathrm{s}^{\prime}$, we replace the $\mathrm{s}^{\prime}$ with $\mathrm{s}^{*^{\prime}}$. The proposed ILS algorithm stops when the time limit is reached. For a local search algorithm, the definition of the neighborhood plays an important role. In our ILS algorithm, we proposed a new neighborhood called Block Exchange, which has never been considered for the single machine scheduling to our best knowledge. At the same time, in order to evaluate the neighborhood solutions in an efficient way, we employ a fast evaluation strategy. The perturbation process is to randomly execute the Block Exchange for the current job sequence. In the following, we present the Block Exchange neighborhood and the fast evaluation strategy of the ILS algorithm in detail.

\section{Block Exchange neighborhood structure}

In this paper, we propose a new neighborhood structure, called Block Exchange, which is composed of all the feasible neighborhood solutions after Block Exchanges in solving $1 \| \sum w_{j} T_{j}$ problem. In author's previous study [6], a new neighbourhood structure called Block Move was proposed to solve single machine scheduling problem with setup times. Block Move and Block Exchange have some similarities and differences. For example, they both are operating on the Blocks, and Block Move is to move one Block to the other position, while Block Exchange is to exchange two Blocks' positions.

The Block Exchange is defined as follows: in the current job sequence $\pi=\left\{\pi_{1}, \pi_{2}, \pi_{3}, \ldots, \pi_{N}\right\}$, exchange two $l$ consecutive jobs with the start positions of $m$ and $n$, respectively (the $l$ consecutive jobs are called block, Block). The range of $m, n$, and $l$ are $m<n, 1 \leq m \leq N-2 l+1, m+l \leq n \leq N-l+1,1 \leq l \leq N / 3$, respectively. The Block Exchange can be simply denoted as Block Exchange $(m, n, l)$. For example, the Block Exchange $(3,10,4)$ is performed in a job sequence of 15 jobs, the process of which is shown in Fig 1.

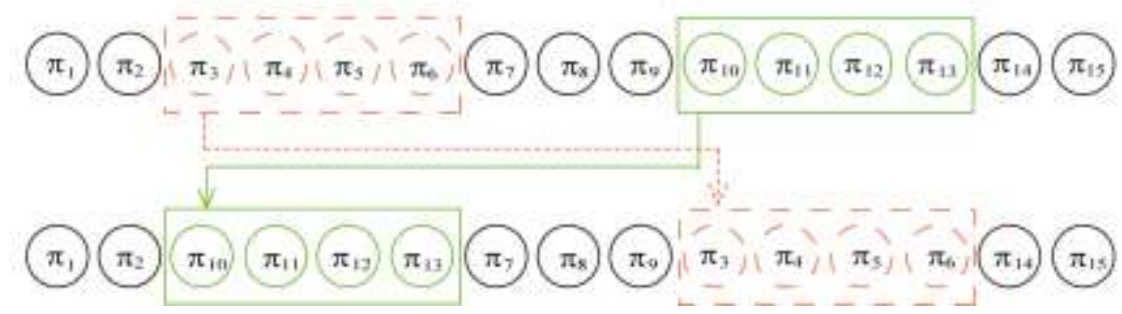

Fig. 1: process of the Block Exchange $(3,10,4)$

In the previous papers for the single machine scheduling, the other neighbourhoods, swap and 2-edge exchange, were employed in [7], which are special cases of our Block Exchange neighborhood where $l=1$ and 2 , respectively.

\section{Fast Evaluation Strategy for the Block Exchange neighborhood}

The evaluation of the neighborhood solution needs the biggest amount of computation time in the local search algorithm. The efficient evaluation strategy can not only improve the efficiency of the algorithm, but also improve the probability of finding the better solution. In this paper, based on the strategy of the Block Move neighborhood structure and the fast incremental evaluation technique proposed by Xu et al. [6] in solving the single machine scheduling with sequence-dependent setup times, a new Block Exchange 
neighborhood structure and its fast evaluation strategy are proposed to solve the single-machine scheduling problem without setup times. The main idea is that for the job sequence $\pi$, the new job sequence $\pi^{\prime}$ is obtained after carrying out the Block Exchange. There is increment $\Delta$ between the jobs affected in job sequence $\pi$ and $\pi$ '.

Calculated the increment $\Delta f\left(\pi_{j}\right)$ of the jobs $\pi_{j}$ affected by Block Exchange and obtained the objective function value $\Delta f(\pi)$ of the new job sequence $\pi^{\prime}$ by using the sum of $\Delta f\left(\pi_{j}\right)$. In this way, the computational complexity can be greatly reduced and achieving a fast evaluation strategy for the Block Exchange neighborhood solutions.

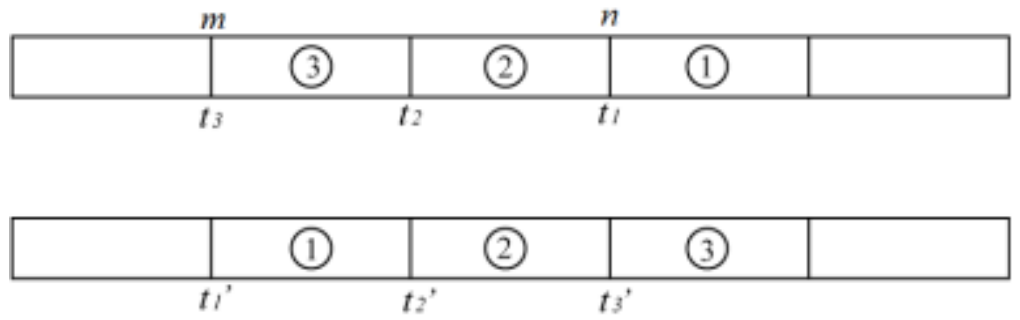

Fig. 2: illustration of the fast evaluation strategy for Block Exchange (m, n, 1)

Taking the Block Exchange ( $m, n, l)$ as an example to illustrate the fast evaluation strategy proposed in this paper. As shown in Fig. 2, after the Block Exchange $(m, n, l)$, the affected jobs are included in the blocks (1), (2), and (3) $\mathrm{t} 1$ represents the start time of the Block (1), the first position and the length of which are $n$ and $l$; 2 represents the start time of the Block (2), the first position and the length of which are $m+l$ and $n-m-l$; t3 represents the start time of the Block (3), the first position and the length of which are $m$ and $l$.

t1', t2', and t3' represent the start times of the Blocks (1), (2), and (3) after executing Block Exchange ( $\mathrm{m}, \mathrm{n}, 1$ ), respectively. $\Delta 1, \Delta 2$, and $\Delta 3$ denote the time increments of Blocks (1), (2), and (3) after the Block Exchange (m, $\mathrm{n}, \mathrm{l})$, respectively, and the formula of which are as follows:

$\Delta 1=t 1^{\prime}-t 1 ; \Delta 2=t 2^{\prime}-t 2 ; \Delta 3=t 3^{\prime}-t 3$

The detailed calculation procedures of $\Delta 1, \Delta 2$, and $\Delta 3$ are as follows:

$$
\begin{aligned}
t 1 & =C_{\pi_{(n-1)}} \\
t 2 & =C_{\pi_{(m+l-1)}} \\
t 3 & =C_{\pi_{(m-1)}} \\
t 1^{\prime} & =C_{\pi_{(m-1)}} \\
t 2^{\prime} & =\Delta 1+C_{\pi_{(n+l-1)}} \\
t 3^{\prime} & =\Delta 2+C_{\pi_{(n-1)}} \\
\Delta 1=t 1^{\prime}-t 1 & =C_{\pi_{(m-1)}}-C_{\pi_{(n-1)}} \\
\Delta 2=t 2^{\prime}-t 2 & =\Delta 1+C_{\pi_{(n+l-1)}}-C_{\pi_{(m+l-1)}} \\
\Delta 3=t 3^{\prime}-t 3 & =\Delta 2+C_{\pi(n-1)}-C_{\pi_{(m-1)}}
\end{aligned}
$$

(1) As shown in Fig. 2, after the Block Exchange $(m, n, l)$ on the job sequence $\pi$, the value of time increments $\Delta$ of jobs $\pi_{j}$ varies according to the location of $\pi_{j}$ :

When the job $\pi_{j}$ is located in the Block (1), $j \in[n ; n+l-1], \Delta=\Delta 1$;

When the job $\pi_{j}$ is in the Block (2), $j \in[m+l ; n-1], \Delta=\Delta 2$; 
When the job $\pi_{j}$ is located in the Block (3), $j \in[m ; m+l-1], \Delta=\Delta 3$.

(2) As shown in Fig. 2, the affected job $\pi_{j}$ after the Block Exchange $(m, n, l)$ on job sequence $\pi$, $j=\{m, \ldots, n+l-1\}$. For the job $\pi_{j}, \Delta f\left(\pi_{j}\right)$ is considered in the following cases:

When the completion times of the job $\pi_{j}$ before and after the Block Exchange $(m, n, l)$ are both less than the due date, i.e., $C_{\pi_{j}} \leq d_{\pi_{j}}$ and $C_{\pi_{j}}+\Delta \leq d_{\pi_{j}}$.The weighted tardiness of job $\pi_{j}$ before and after the Block Exchange operation both are zero, i.e., $\Delta f\left(\pi_{j}\right)=0$.

When the completion time of the job $\pi_{j}$ before and after the Block Exchange $(m, n, l)$ both are more than the due time, i.e., $C_{\pi_{j}}>d_{\pi_{j}}$ and $C_{\pi_{j}}+\Delta>d_{\pi_{j}}$, then $\Delta f\left(\pi_{j}\right)=w_{\pi_{j}} \Delta$.

When the completion time of the job $\pi_{j}$ before the Block Exchange $(m, n, l)$ is less than the due date, i.e., $C_{\pi_{j}} \leq d_{\pi_{j}}$, and the completion time after the Block Exchange $(m, n, l)$ is more than the due date, i.e., $C_{\pi_{j}}+\Delta>d_{\pi_{j}}$, then $\Delta f\left(\pi_{j}\right)=w_{\pi_{j}}\left(C_{\pi_{j}}+\Delta-d_{\pi_{j}}\right)$.

When the completion time of the job $\pi_{j}$ before the Block Exchange $(m, n, l)$ is more than the due date, i.e., $C_{\pi_{j}}>d_{\pi_{j}}$, the completion time after the Block Exchange $(m, n, l)$ is less than the due date, i.e., $C_{\pi_{j}}+\Delta \leq d_{\pi_{j}}$, then $\Delta f\left(\pi_{j}\right)=-w_{\pi_{j}}\left(C_{\pi_{j}}-d_{\pi_{j}}\right)$.

(3) Find the value $\Delta f\left(\pi_{j}\right), j=\{m, \ldots, n+l-1\}$, and calculate $\Delta f(\pi)=\sum_{j=m}^{n+l-1} \Delta f\left(\pi_{j}\right)$. And the objective function value $f\left(\pi^{\prime}\right)$ of the job sequence $\pi^{\prime}$ after the Block Exchange $(m, n, l)$ is obtained, i.e., $f\left(\pi^{\prime}\right)=f(\pi)+\Delta f(\pi)$, in which $f(\pi)$ is the objective function value of the job sequence $\pi$.

\section{Computational results}

\subsection{Experiment scheme}

In order to show the effectiveness of the newly proposed Block Exchange neighborhood and the fast evaluation strategy, we compare them with two previously used neighborhoods, swap and 2-edge exchange. Thus, three ILS algorithms, denoted by ILS_BE, swap, and 2-edge, are designed, which adopt the Block Exchange neighborhood and fast evaluation strategy, swap neighbourhood, and 2-edge exchange neighborhood, respectively.

The benchmark data of the $1 \| \sum w_{j} T_{j}$ problem widely used in the literature consisted of 125 problem instances, each with 40 jobs [8]. The three ILS algorithms, ILS_BE, swap, and 2-edge, both are programmed in $\mathrm{C}++$ and compiled on a PC using Linux with $2.6 \mathrm{GHz}$ CPU and $64.0 \mathrm{~Gb}$ RAM. For the three ILS algorithms, we solved each problem instance independently for 100 times using different random seeds, subject to a time limit of $50 \mathrm{CPU}$ seconds.

\subsection{Comparisons with other two neighborhoods}

We summarize the comparisons of three ILS algorithms, swap, 2-edge, and ILS_BE, with the bestknown results for the 125 instances in Table 1, in which the numbers of better, equal, and worse solutions compared with the best-known results are presented.

Table 1.summary of comparing the results of three ILS algorithms with the best-known results

\begin{tabular}{|l|l|l|l|}
\hline & swap & 2-edge & ILS_BE \\
\hline number of improved solutions & 0 & 0 & 0 \\
\hline number of equal solutions & 33 & 28 & 125 \\
\hline number of inferior solutions & 92 & 97 & 0 \\
\hline number of all solutions & 125 & 125 & 125 \\
\hline
\end{tabular}

In Table 1, we observe that ILS_BE algorithm is quite competitive in terms of this criterion. Comparing the results of three ILS algorithms, swap, 2-edge, and ILS_BE, with the best-known results, the numbers of 
equal solutions were 33, 28, and 125, respectively. And the numbers of worse solutions were 92, 97, and 0, respectively. From Table 1, we conclude that the ILS_BE algorithm outperforms the other two ILS algorithms in terms of solution quality. These results show the efficacy of the proposed Block Exchange neighborhood and its fast evaluation strategy.

\section{Conclusion}

In this paper, a new neighborhood structure Block Exchange and its fast evaluation strategy are proposed to minimize the total weighted tardiness when using the ILS algorithm to solve the $1 \| \sum w_{j} T_{j}$ problem. The Block Exchange neighborhood expands the search space, while the fast evaluation strategy improves the search efficiency to reduce the calculation time. From the comparisons of experiments, it is proved that the Block Exchange neighborhood and the fast evaluation strategy can improve the efficiency and find the better solutions in a certain extent. Although this paper mainly discusses the single machine scheduling, it provides the new ideas to the complex scheduling problem and other combinatorial optimization problem, which has certain research significance.

\section{Acknowledgments}

The research was supported by the Doctoral Scientific Research Foundation of JiangHan University, WuHan (Grant no. 1008-06280004).

\section{References}

[1] Lawler E L. A "pseudopolynomial” algorithm for sequencing jobs to minimize total tardiness. Annals of discrete Mathematics, 1977, 1:331-342

[2] Lenstra J K, Rinnooy Kan A, Brucker P. Complexity of machine scheduling problems. Annals of discrete mathematics, 1977, 1:343-362

[3] Tasgetiren MF, LiangYC, SevkliM, Gencyilmaz G. Particle swarm optimization and differential evolution for the single machine total weighted tardiness problem. Int J Prod Res 2006; 44(22): 4737-54.

[4] Ü Bilge, Kurtulan M, Kıraç F. A tabu search algorithm for the single machine total weighted tardiness problem. Eur J Oper Res2007; 176:1423-35.

[5] Wang X, Tang L. A population-based variable neighbourhood search for the single machine total weighted tardiness problem. Comput Oper Res 2009; 36: 2105-10.

[6] Xu H, Lü Z, Cheng T C. Iterated Local Search for single-machine scheduling with sequence-dependent setup times to minimize total weighted tardiness[J]. Journal of Scheduling, 2014, 17(3):271-287.

[7] Kirlik, G., \& Oguz, C. (2012). A variable neighbourhood search for minimizing total weighted tardiness with sequence dependent setup times on a single machine. Computers \& Operations Research, 39(7), 1506-1520.

[8] Potts C N, Wassenhove L N V. A Branch and Bound Algorithm for the Total Weighted Tardiness Problem[J]. Operations Research, 1985, 33(2):363-377. 